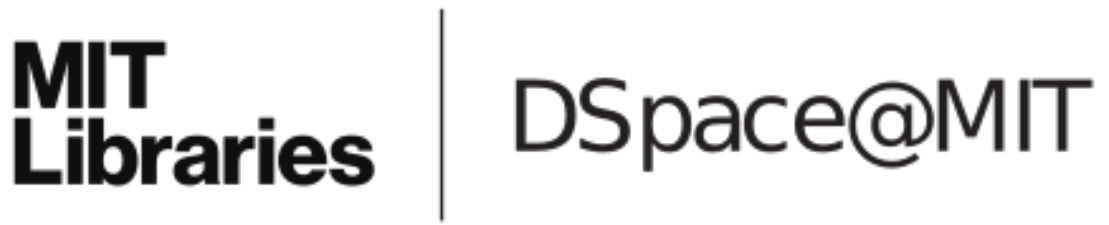

\author{
MIT Open Access Articles
}

Revisiting the Physics of Spider Ballooning

The MIT Faculty has made this article openly available. Please share how this access benefits you. Your story matters.

Citation: Sheldon, K.S. et al. "Revisiting the Physics of Spider Ballooning." Women in Mathematical Biology 8 (August 2017): 163-178 ( 2017 The Author(s) and the Association for Women in Mathematics

As Published: http://dx.doi.org/10.1007/978-3-319-60304-9_9

Publisher: Springer International Publishing

Persistent URL: https://hdl.handle.net/1721.1/125314

Version: Author's final manuscript: final author's manuscript post peer review, without publisher's formatting or copy editing

Terms of use: Creative Commons Attribution-Noncommercial-Share Alike 


\title{
Revisiting the physics of spider ballooning
}

Kimberly S. Sheldon, Longhua Zhao, Angela Chuang, Iordanka N. Panayotova, Laura A. Miller and Lydia Bourouiba

\begin{abstract}
Spiders use a unique type of aerial dispersal called ballooning to move from one location to another. In order to balloon, a spider must first release one or more flexible, elastic, silk draglines from its spinnerets. Once enough force is generated on the dragline(s), the spider becomes airborne. This take-off stage of ballooning is followed by the flight stage and finally the settling stage when spiders land in a new location. Though the ecology of spider ballooning is well understood, little is known about the physical mechanisms. This is in part due to the significant challenge of describing the relevant physics for spiders that are ballooning across large distances. One difficulty, for example, is that properties of both the spider, such as body size and shape, and the silk dragline(s) can vary among species and individuals. In addition, the relevant physics may differ among the three stages of ballooning. Finally, models must take into account the interaction between the flexi-

Kimberly S. Sheldon

Department of Ecology and Evolutionary Biology, University of Tennessee, Knoxville, TN 37996, USA, e-mail: ksheldon@utk.edu

Longhua Zhao

Department of Mathematics, Applied Mathematics and Statistics, Case Western Reserve University, 10900 Euclid Avenue, Cleveland, OH 44106, USA e-mail: longhua.zhao@case.edu

Angela Chuang

Department of Ecology and Evolutionary Biology, University of Tennessee, Knoxville, TN 37996, USA e-mail: angelachuang@utk.edu

Iordanka N. Panayotova

Department of Mathematics, Christopher Newport University, Newport News, VA 23606, USA e-mail: iordanka.panayotova@cnu.edu

Laura A. Miller

Departments of Biology and Mathematics, Phillips Hall, CB \#3250, University of North Carolina, Chapel Hill, NC 27599-3280, USA e-mail: lam9@email.unc.edu

Lydia Bourouiba

The Fluid Dynamics of Disease Transmission Laboratory, Massachusetts Institute of Technology, Cambridge, MA 02130, USA e-mail: lbouro@mit.edu
\end{abstract}


ble dragline and air, and resolving this multi-scale, fluid-structure interaction can be particularly difficult. Here, we review the literature on spider ballooning, including the relevant physics, meteorological conditions that favor ballooning, and previous mechanical models used to describe ballooning. We then highlight challenges and open questions relevant to future modeling of spider ballooning.

\section{Ecology of spider dispersal}

Dispersal, or the movement of individuals from their birth location to a new location [10], is a critical stage in the life cycle of many species. The benefits of dispersal include reduced competition for resources (e.g. reproductive mates and food) and inbreeding avoidance $[11,30,48]$, which may ultimately lead to greater survival and reproduction.

Given the importance of dispersal for reproductive success, species have evolved a variety of mechanisms to move to new locations. One such mechanism that can be observed in spiders (Araneae), is a type of aerial dispersal called 'ballooning' $[5,16]$. To become airborne via ballooning, a spider first climbs to a high point and initiates a 'tiptoe' posture in which it stands on the ends of its tarsi and elevates its abdomen (Figure 1). The spider then releases one or more silk lines, or draglines, from its spinnerets [38]. With enough force on the dragline(s), the spider is lifted and becomes airborne [36, 38]. This 'take-off' stage is followed by the 'flight' stage in which individuals can travel as high as $5 \mathrm{~km}$ above ground [15] and as far as 3200 $\mathrm{km}$ in distance [23]. Finally, spiders reach the 'settling' stage when they land in a new location.

Because adult spiders can have hundreds of spiderlings - or young spiders - from a single reproductive event, ballooning allows spiderlings to avoid competition and cannibalism from siblings and conspecific adults [44]. Though some species of spider are known to balloon at any time of year and any phenological stage [45], other species balloon only during specific time periods and clearly defined stages [13]. Regardless of when they disperse, ballooning spiders rarely have a mass greater than $100 \mathrm{mg}[46,38]$ (but see [34] for exception), which suggests constraints on body size for aerial dispersal. In addition, wind speeds for take-off appear to always be below $3 \mathrm{~m} / \mathrm{s}$ [44].

Despite all we know about ballooning and its importance in the ecology and evolution of spiders, the physical mechanisms related to this unique type of dispersal are still poorly understood. Generating a model for spider ballooning seems simple in practice, but it is a complex problem given the variety of parameters involved. Ballooning is used by a host of species that come in different shapes and sizes. Both inertial and viscous forces in the surrounding air are important. Models must also consider dragline properties, including number of threads, length, elasticity, electrostatics, and compliance as well as constraints on wind speed [44] and spider mass $[46,38]$. After incorporating all of the various parameters, one is left with 
a host of possible regimes, and different regimes could dominate during the three stages of spider ballooning (i.e. take-off, flight, and settling).

Here, we review the existing literature on the physics of this fascinating mode of ecological dispersal and identify areas for future research. We first examine the relevant physics related to ballooning. We then discuss previous analytic models that researchers have used to parse out the dominant regimes. Finally, we highlight the remaining questions surrounding spider ballooning and how future research might address these questions.

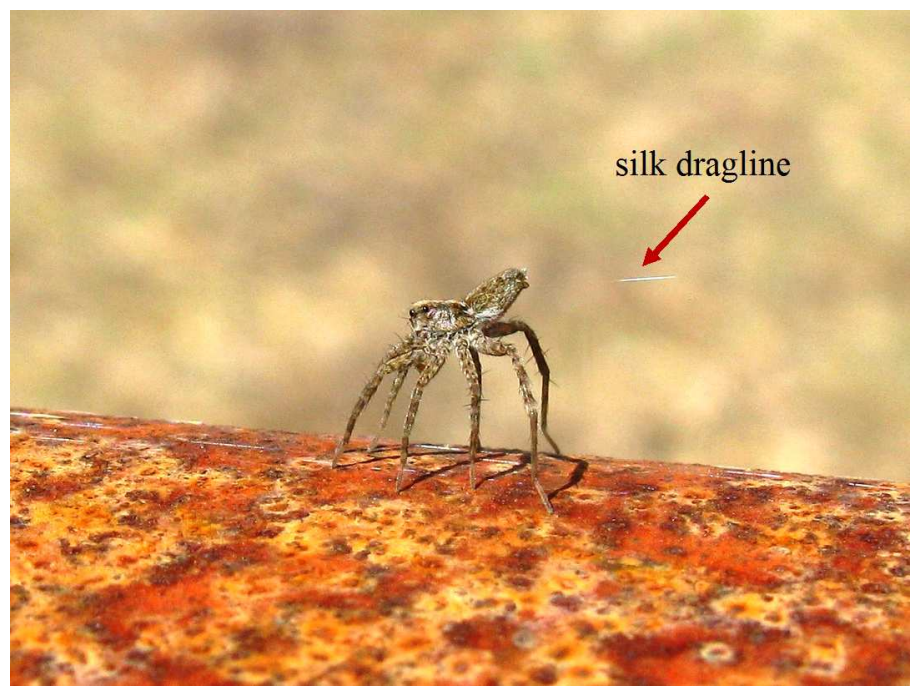

Fig. 1 Prior to ballooning, spiders display a tiptoe behavior which involves standing on the ends of the tarsi, angling the abdomen upward, and releasing one or more silk draglines from spinnerets. (c) Sarah Rose.

\section{Relevant physics}

\subsection{The physical parameter space}

The parameters relevant to models of spider ballooning can be broadly characterized as those relating to individual morphology, dragline characteristics, and ambient environmental conditions. Individual morphology can be a constraining factor in flight [27], where mass determines the amount of vertical drag force required to lift off and sustain flight. Due to upper limits in dragline tensile strength and ambient air velocity, larger masses may completely preclude take-off for individuals $[38,41]$. 
This can partially explain why ballooning as a dispersal mechanism is typically observed in younger instars and smaller taxa [3].

Body length and shape influence the amount of drag generated to sustain flight. Despite the large variation in body shapes among spider taxa that balloon (Figure 2), all previous work on spider ballooning has used a spherical shape to model the spider $[27,31,32]$. Post-release posturing of the legs may have additional implications for terminal velocities [38], but the effects of a spider's eight legs on drag and flight speed have thus far been ignored.

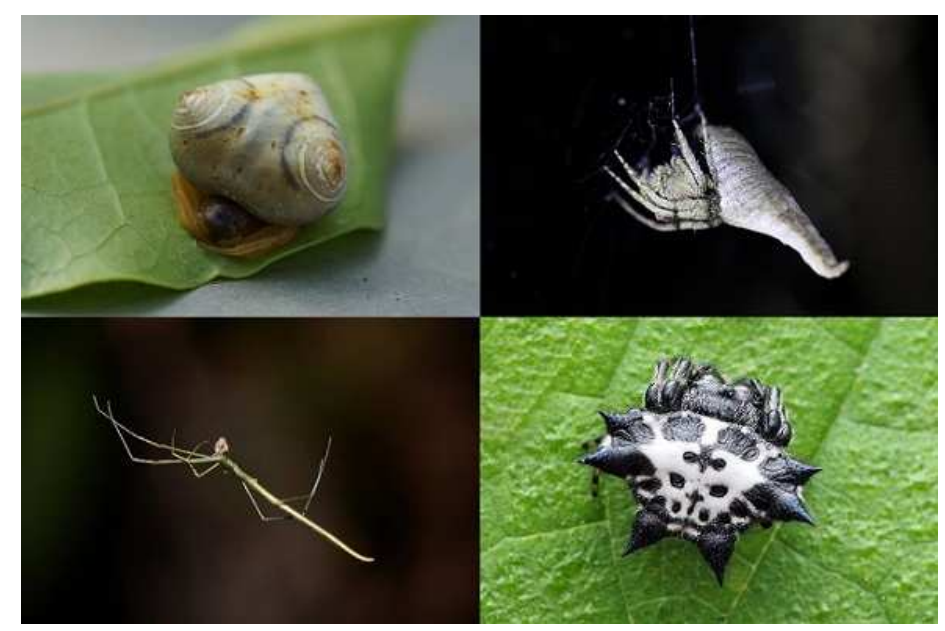

Fig. 2 Spiders may display unique body shapes that cannot be realistically modeled as a sphere, including spiders in the genera (clockwise from the top left) Cyrtarachne, Poltys, Gasteracantha, and Ariamnes. Photo credit to Yung Yi Tsai, Fisher Chen, Robert Tsai, Sun Jong Liu.

The physical characteristics of the dragline act together with the body to create surfaces for aerodynamic drag to be generated. Dragline mass is generally considered negligible in models since it is minuscule compared to the spider's body mass. This is true even in cases where an individual may release two or more dragline threads, as has been observed across multiple ballooning taxa [14, 34]. Unlike mass, dragline length has been shown to be important in take-off dynamics and in decreasing terminal velocity $[2,37]$, but its influence during flight and on dispersal distance has been debated [31]. Because silk can flex and extend [31], distortion and bending during flight can decrease the effective length of the dragline. Interestingly, however, observations of entangled draglines during ballooning have not been reported.

Dragline flexibility influences the spider's movement through space. Since the dragline bends and adapts to flow structures in the air, it subjects the attached spider to variations in flow profiles. Thus, factors that influence dragline flexibility, including elasticity, stiffness, bending rigidity, and flexural rigidity, should be included in models. 
Another important characteristic to consider when modeling ballooning is the electrostatic properties of silk [28]. Electrically charged silk may explain how a long, single dragline can avoid becoming entangled during flight. In cases where dozens of silken threads are released together, electrostatic repulsion in individual strands may similarly prevent mass entanglement [34]. Electrostatic forces are speculated to act independently from advective and aerodynamic forces on the spider, thus providing additional lift [19].

Lastly, environmental conditions play a substantial role in spider take-off, flight, and landing. Meteorological conditions seem to affect the initiation of tiptoe behavior; multiple field observations suggest that spiders balloon during daylight hours, under sunny and clear skies, and at wind speeds less than $3 \mathrm{~m} / \mathrm{s}$ [13, 27, 22, 44, 38]. Wind speed has strong implications for take-off velocities and distance traveled. Horizontal wind velocity is directly related to the horizontal velocity that ballooning spiders attain [27, 37], whereas vertical wind velocity and the resulting drag generated counteracts the falling speed of the body. Given the small spatial scales in which ballooning spiders operate, minor variations in meteorological conditions can subject individuals to frequent changes in velocity and direction [38].

\subsection{Relevant dimensionless parameters}

Several dimensionless parameters are relevant to models of spider ballooning and can be obtained through scale analysis, which describes fluid flow behavior. Scaling estimates the magnitudes of various forces acting on a body and governing fluid motion. Thus, scale analysis can be used to understand the meteorological state of the atmosphere that favors the initiation and maintenance of spider ballooning.

Initiation of the spider ballooning, or the take-off, could be aided by the presence of thermals. Thermals are parcels of hot air that develop as a result of convection when cold air sinks and hot air rises as shown in Figure 3. The atmospheric conditions that favor thermal formation can be established by examining the Richardson number, which characterizes the relationship between buoyancy and shear forces and is defined by

$$
R i=\frac{N^{2}}{\left(\frac{d \bar{U}}{d z}\right)^{2}}=-\left(\frac{g}{\rho_{0}}\right) \frac{\frac{d \bar{\rho}}{d z}}{\left(\frac{d \bar{U}}{d z}\right)^{2}},
$$

where $\mathrm{g}$ is the gravitational constant, $\bar{\rho}$ is the mean pressure, $\bar{U}$ is the mean horizontal wind velocity, $z$ denotes the vertical coordinate direction, and $N$ is the BruntVaisala frequency or buoyancy frequency. The Richardson number quantifies the ratio of buoyancy-generated to shear-generated instability in the flow field [40].

Observational studies of aerial dispersal of spiders show a strong correlation between the number of spiders caught during dispersal and the Richardson number $[43,41]$. The number of spiders caught in traps was significantly larger when the Richardson number was negative, which characterizes strong, unstable stratification 
and/or weak horizontal wind shear typical of calm, sunny days [43, 21]. This number could be used to describe the overall general conditions of take-off, though it does not relate to the properties of take-off or flight of the spider itself.

Another important dimensionless number relevant to spider ballooning is the Reynolds number. As an object moves through the air, aerodynamic forces between the object and the air are generated. The magnitude of forces depends on the shape, speed, and mass of the object, as well as properties of the fluid, specifically the viscosity, or stickiness, and the density. The Reynolds number relates the inertial forces to the viscous forces and is defined as

$$
\operatorname{Re}=\frac{\rho U L}{\mu}
$$

where $\rho$ and $\mu$ are the density and the dynamics viscosity of the surrounding media (air), respectively, $\mathrm{U}$ is the characteristic velocity, and $\mathrm{L}$ is the characteristic length, which could be either the spider's body length or the length of the dragline [29]. The Reynolds number is used to characterize different flow regimes. $R e<2000$ is typical for laminar flows, and $R e>4000$ characterizes turbulent flows [29]. For spider ballooning, values of $\operatorname{Re}_{D}<10$ (where $D$ is the spider's body length) indicate that the stimulus prompting the ballooning activity is too weak. In contrast, for values of $\operatorname{Re}_{D}>200-400$, it seems that the motion of the wind is too vigorous for the spiders to safely conduct aerial dispersal [27].

A third important dimensionless parameter used to analyze aerodynamic forces is the Froude number. Defined as the ratio of the inertial force to the gravitational force, it is used to quantify the effect of gravity on the moving object. The Froude number is described as $F r=\frac{U}{\sqrt{g L}}$, where $U$ is the wind velocity or the velocity of free fall, $L$ is the characteristic length of the moving object, and, in the case of spiders, $L$ is the diameter of the body. The Froude number has been used in studies of both terrestrial and aquatic locomotion to account for the effect of gravity on movement of arthropods and mammals [4]. It has not, however, been used in relation to spider ballooning. One of the goals of our future research is to use this parameter to evaluate the significance of gravity on the dynamics of spider ballooning.

The last dimensionless number that is important for models of spider ballooning is the Strouhal number $(S t)$, which is defined as $S t=\frac{f L}{U}$, where $f$ is the frequency of vortex shedding (found numerically or experimentally), $L$ is the length of the dragline, and $U$ is the velocity of air relative to the velocity of the dragline (found as a sum of free fall velocity and wind speed). The Strouhal number is frequently used to describe the tail or wing kinematics of swimming or flying animals because it is known to govern a well-defined series of vortex growth and shedding regimes for airfoils undergoing pitching and heaving motions [25], [39]. Propulsion efficiency is high under a narrow range of Strouhal numbers and usually peaks within the interval $0.2<S t<0.4$ [39]. The Strouhal number has not been used in analyses of spider ballooning dynamics. One of our future goals is to evaluate the Strouhal number for the passive locomotion of spider ballooning both numerically and experimentally. 


\section{Meteorological conditions favoring spider ballooning}

Small, negative Richardson numbers are found on calm, sunny days when unstable stratification and/or weak horizontal wind shears dominate. Such meteorological conditions are known to be favorable for spider ballooning and occur close to the Earth's surface in the early morning or late afternoon when temperature convection, or temperature inversion, happens naturally in the atmosphere [1]. The vertical movement of warmer air up and cooler air down results in static instabilities in the atmosphere and leads to the formation of a vertical, turbulent layer filled with vortices, called thermals, that can be used as a lifting force by ballooning spiders.

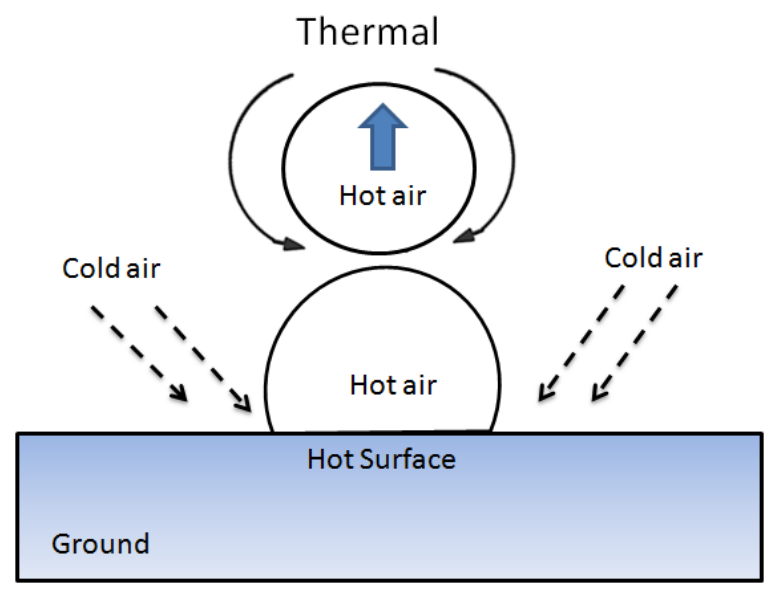

Fig. 3 The development of a thermal, with the rise of hot, lighter air initiating convection.

Greenstone [22] focused on defining meteorological variables related to the production and maintenance of thermals and used them to predict the number of ballooning spiders. The author showed that clear sky meteorological conditions, which are necessary for thermal production, were present in only $82 \%$ of spider ballooning observations. This suggests that another source of uplift for ballooning that can generate vertical velocity may be present. A continuously stratified atmosphere can destabilize if the Richardson number is less than 0.25 (i.e. $0<R i<0.25$ ). KelvinHelmholtz instability can take place as shown in Figure 4 and vorticies that grow in amplitude can lead to turbulence and vertical mixing as well [12]. However, a recent study showed that the timing of spider ballooning is not purely dominated by convection as some horizontal shear is also present. [32].

The Kelvin-Helmholtz instability is only one particular type of instability that can affect spider ballooning. A zone with a wind shear, or a sudden change in the wind's speed or direction, is a well-known source of turbulence. The shearing, which can be horizontal, vertical, or both, creates forces that produce eddies along the mixing zone. The formed eddies may range in diameter from a couple of meters to several 
hundred meters [1]. Shear layers are a major cause of turbulence and, if they are formed high in the atmosphere, can often produce clear-air turbulence.

\section{Kelvin-Helmholtz Instabilities}

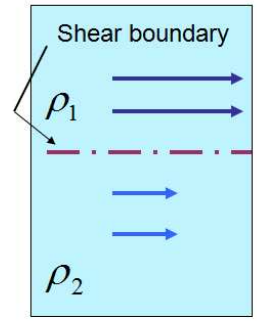

Increasing shear

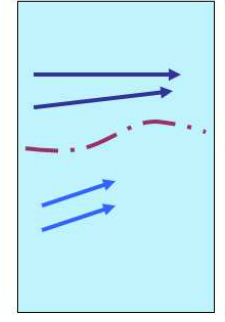

Boundary deforms

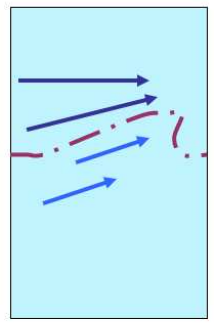

Waves appear

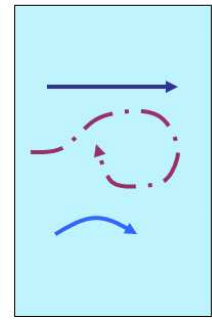

Turbulent eddies break

Fig. 4 Kelvin-Helmholtz shear instability resulting in the formation of turbulent eddies and mixing.

Shear layers can also be found near the Earth's surface, where turbulent whirling eddies are formed due to the roughness of the ground. As wind blows over a landscape full of obstacles such as trees and mountains, it breaks into irregular air motions known as wind gusts or turbulent eddies. The size and shape of these eddies depend on the dimensions of obstacles and the wind speed and can influence the air flow for hundreds of meters above the surface [1].

Another source of eddies are orographic perturbations, which occur when strong winds blow perpendicular to mountain ranges. Air flowing over the top of a mountain produces perturbations when it reaches the other side. The associated updrafts and downdrafts formed on the leeward side of the mountain may extend to heights from 2 to 20 times the height of the mountain peaks [1]. All of the meteorological scenarios summarized above may produce turbulence and/or eddies and may serve as a driving force for spider lift and subsequent dispersal.

\section{Previous mechanical models for ballooning}

\subsection{Humphrey's take-off model}

The first analytical model for spider ballooning was built by Humphrey [27] who studied the phenomenon with a simple fluid mechanical model (see figure 5 (A)). In Humphrey's model, the spider was represented as a solid sphere. Attached to the solid sphere was a rigid, inextensible, cylindrical rod that was used to approximate a silk dragline, thus producing a "lollipop" appearance. The rod was considered to be massless relative to the spider. 


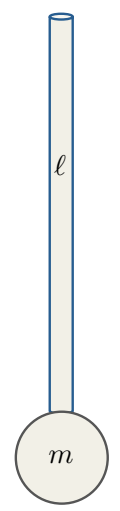

(A) Humphrey's model

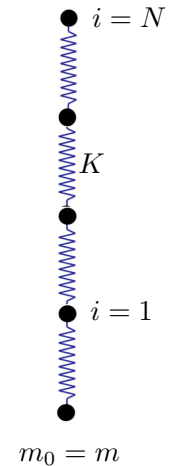

(B) Reynolds et al.'s model

Fig. 5 Schematic of previous mechanical models of spider ballooning. In (A) Humphrey represented the spider as a solid sphere $(m)$ attached to a rigid, inextensible, cylindrical, slender rod $(\ell)$ that approximates a dragline. The rod is considered to be massless relative to the sphere. In (B) Reynolds et al. represented the spider as a mass point $m_{0}$ attached to a flexible dragline made of a chain of $N$ springs with spring constant $K$. The mass of the dragline is distributed on the nodes along the chain.

For the lollipop model, the governing equation of the model was a balance of inertia, buoyancy, frictional and pressure forces:

$$
\begin{aligned}
\rho_{s} V_{s} \frac{d \mathbf{U}}{d t}= & -\rho \chi V_{s} \frac{d(\mathbf{U}-\mathbf{V})}{d t}-\alpha|\mathbf{U}-\mathbf{V}|(\mathbf{U}-\mathbf{V}) \\
& +\left(\rho-\rho_{s}\right) V_{s} \mathbf{g}+\rho V_{s} \frac{d \mathbf{V}}{d t}+B(t)
\end{aligned}
$$

where $\rho_{s}$ and $V_{s}$ are the sphere's density and volume, respectively, $\alpha$ and $\mathbf{U}$ are the sphere-rod drag coefficient and velocity, respectively, $\mathbf{V}$ is the fluid (air) velocity, $\rho$ is the density of fluid (i.e., air), $\chi$ is the coefficient of added mass ( 0.5 for a sphere), $\mathbf{g}$ is the gravity vector, and $B(t)$ is the Basset term. Both added fluid mass and Basset force are due to the unsteadiness of the problem and the author ended up neglecting the Basset force in the simulation. Equation (3) was modified from previous research $[26,6]$ in which $\alpha$ was not specified for a sphere-rod system. A range of Reynolds numbers was defined based on wind velocity, spider length and mass. For the drag coefficients for silk, slender bodies in low Reynolds number regimes were taken from Happel and Brenner [24]. The equations for the drag coefficient for the sphere were empirical correlations reported by Clift et al. [6]. However, the drag expression used to obtain the vertical take-off velocity of the spider was that of the turbulent regime:

$$
m g=C_{d} d \ell \frac{1}{2}\left(V_{z}\right)_{\min }^{2}
$$

where $m$ is the mass of the spider, $g$ is the acceleration due to gravity, $C_{d}$ is the filament drag coefficient, $d$ is the filament diameter, $\ell$ is the filament length, and $\left(V_{z}\right)_{\min }$ 
is the minimum vertical component of air velocity required to initiate ballooning. In this model, drag determines environmental conditions necessary for take-off and is highly dependent on the dragline length. Numerical simulations were used to examine the spider-filament free fall in a quiescent environment and in sinusoidally oscillating vertical wind with average background wind vertical velocity of $\bar{V}_{z}=0$. The minimum vertical wind required to initiate ballooning and the distance travelled over time were reported as relations of spider mass and dragline length.

Humphrey's results were supported by empirical investigations of drag on spiders and their silk by Suter $[36,37,38]$. However, the physical properties and dimensions in Humphrey's model were not validated. In particular, the numerical simulation of the system of Ordinary Differential Equations (Eqn. 1.3) for the simple lollipop model suggested that spiders with short silk draglines in strong winds travel faster and farther than the same sized spider with a long dragline in weaker winds. This contradicts the observation that spiders usually balloon in $<3 \mathrm{~m} / \mathrm{s}$ winds. Finally, Humphrey's lollipop model suggested that a group of small spiders on a silk dragline would travel faster and farther than a single spider of equivalent mass on the same dragline and in the same wind conditions.

\subsection{Reynolds et al.'s passive dispersal model}

Instead of the rigid rod used in Humphrey's model [27], Reynolds et al. [31, 32] modeled the silk dragline as a chain of springs and spheres that resist stretching but not bending (Fig. 5 (B)). Passive dispersal of the flexible silk filament was then modeled in turbulent flow that approximated the atmospheric boundary layer. Similar to Humphrey's work [27], take-off dynamics were not described. The Reynolds et al. model [31] described the force $\mathbf{F}_{i}$ acting on each node $i=0, N$ as follows:

$$
\mathbf{F}_{i}=m_{i} \tau_{s}^{-1}\left(\mathbf{u}_{i}-\mathbf{v}_{i}\right)+K\left(\mathbf{p}_{i, i-1} s_{i, i-1}+\mathbf{p}_{i, i+1} s_{i, i+1}\right)+m_{0} \mathbf{g} \delta_{i, 0},
$$

where $\mathbf{u}$ is the local air velocity, $m_{0}$ is the spider mass, $\mathbf{v}_{0}$ is the velocity located at node $i=0, m_{i}$ and $\mathbf{v}_{i}$ are mass and velocities at nodes $i=1, \cdots, N$ along the chain, and $K$ is the spring constant. $s_{i, j}$ are differences between the spring lengths to the fixed rest lengths from node $i$ to node $j \cdot \mathbf{p}_{i, i+1}$ are unit vectors orientated along the segment joining the $i$ node and the $i+1$ node. $\tau_{s}$ is the aerodynamic response time of the dragline, and $\delta_{i, 0}$ is the Kronecker delta. This model reflects the assumption of massless spring and that gravity only acts on the node $i=0$ (i.e. the body of the spider).

In a subsequent Reynolds et al. paper [32], the simplistic model of turbulence used in the first Reynolds et al. simulation [31] was extended to a Lagrangian stochastic model. Lagrangian stochastic models for the velocity (u) and position $(\mathbf{x})$ are in the following form 


$$
\begin{aligned}
\frac{d u_{i}}{d t} & =a_{i}(\mathbf{x}, \mathbf{u}, t)+\sqrt{C_{0} \varepsilon} \frac{d \xi_{i}}{d t}, \\
\frac{d \mathbf{x}}{d t} & =\mathbf{u},
\end{aligned}
$$

where the indices $i=1,2,3$ denote Cartesian directions, $C_{0}=5$ is the Kolmogorov's Lagrangian velocity structure constant, $\varepsilon$ is the mean rate of dissipation of turbulent kinetic energy divided by the density of air, $\xi$ is the Wiener white noise, and $a$ is a solution of the Fokker-Plank equation. This approach considered a simple model of turbulence.

Contrary to Humphrey's results [27], Reynolds found that the length of a dragline did not affect dispersal distance [31]. With the flexible dragline model, the condition for maximum dispersal distance was found to be a gradient Richardson number of approximately -3.2 [32]. This model showed that dispersal over several hundred kilometers was possible. However, based on the flexible dragline model, silk could be-

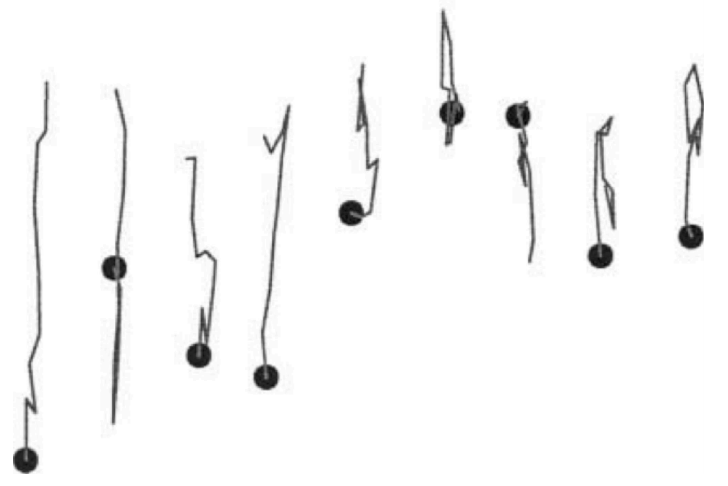

Fig. 6 The entanglement of the silk dragline predicted by the Reynolds et al. model [31]. This figure was reprinted from [31] with permission. come entangled (Fig. 6). Inter-

estingly, experimental studies report that the silk dragline does not become entangle or clump in this manner once the spider is in flight [34].

\subsection{Thomas et al.'s diffusion model}

Instead of using a turbulence model that approximates the atmospheric boundary layer [32], Thomas et al. [42] built a one-dimensional diffusion model,

$$
\frac{\partial n}{\partial t}=D_{s} \frac{\partial^{2} n}{\partial x^{2}},
$$

to capture evolution of the spatial and temporal number of ballooning spiders $n(x, t)$. The solution to the diffusion equation (7) is [7]

$$
n(x, t)=\frac{n_{0}}{2} \operatorname{Erfc}\left(\frac{x}{2 \sqrt{D_{s(t)} t}}\right),
$$

where $n_{0}$ is the initial number of spiders at position $x_{0}, n$ is the number of spiders at position $x$ at time $t, D_{s(t)}$ is the diffusion coefficient of the species $s$ at time $t$, and 
Erfc is the complementary error function. Based on the distribution of spiders in space and time, Thomas and colleagues estimated considerable horizontal distance traveled by the ballooning spider on the order of up to $90 \mathrm{~km}$ in $8 \mathrm{~h} \mathrm{[41]}$

\subsection{Models incorporating electrostatics}

Electrical charging of the spider's dragline is believed to create electrostatic repulsion both within and among the silk filaments [28], allowing filaments to avoid the type of entanglement shown in Fig. 6. Researchers have suggested that, combined with aerodynamic force, the negative surface charge density of the Earth may play a role in spider ballooning. Though meteorological conditions are often believed to be the dominant driver in the take-off stage of spider ballooning, Gorham [19] conjectured that the electrostatic field is a necessary condition for take-off. In Gorham [19], an exponential approximate model for the atmospheric electric field is assumed to be

$$
E(h)=E_{0} e^{-\alpha h}\left(V m^{-1}\right),
$$

where $h$ is height, $\alpha$ is the exponential rate for the exponential fit to the electric field (i.e. $\alpha=3.0 \times 10^{-4} \mathrm{~m}^{-1}$ ), and $E_{0}$ is the electric field at h=0 (i.e. $E_{0}=-120 \mathrm{Vm}^{-1}$ ). Assuming a constant acceleration for a single dragline in a pure electrostatic field, the charge required for take-off is computed as

$$
Q_{\text {accel }}=m\left(a_{\text {net }}+g\right) / E_{0} \approx 100(n C),
$$

where $\mathrm{m}$ is the mass of the spider and an initial net vertical acceleration is in the range of $a_{\text {net }}=3 \sim 6 \mathrm{~ms}^{-2}$. The author concluded that spiders use electrostatic forces only to lift them into the air [19]. Electrostatics may account for some unexplained ballooning phenomena, such as high velocity ballooning in conditions of little or no wind or the observation made by Charles Darwin that spiders were ballooning from his ship, The Beagle, in horizontal movements [8, 19]. However, the role of electrostatics may not be significant since empirical research has shown that spiders are using rising thermals on hot days without wind [34] and, thus, thermal currents could provide all the necessary lift for ballooning.

\section{Challenges, open questions, and needs}

Describing the relevant physics for models of spiders that are ballooning across large distances in turbulent flows presents significant challenges. The material properties of spider silk are complex and can vary among species, individuals, and even within the same organism $[20,17]$. The interaction between the flexible dragline and air must be taken into consideration, and resolving this fluid-structure interaction can be particularly difficult. 
Spider ballooning is also an inherently multiscale problem. When zooming into the flow around an individual spider, turbulence can be handled explicitly using direct numerical simulation. When considering dozens of organisms within largescale geophysical flows, it is necessary to use turbulence models such as Reynolds averaged Navier-Stokes equations (RANS) [47, 18] or large eddy simulations [35, 9,33 . Both methods average out either the entire or the sub-grid flow structure for computational efficiency. However, the details of the flow near the spider and the dragline are critical to resolving the silk's reconfiguration. The development of new multiscale methods, such as hybrid direct numerical simulation / RANS models, may be required to resolve both the viscous flow near the dragline and the atmospheric turbulent flows.

In addition to resolving the flow near the elastic dragline, there are challenges to modeling the atmospheric processes relevant to spider ballooning. The vertical ascent and descent of the spider is likely sensitive to short-term turbulent variability. Mathematical models that average velocities across relatively large distances and over long time intervals may not be appropriate since these simplifications can effectively smooth strong nonlinearities relevant to take-off and flight. On the other hand, resolving such details can quickly make numerical simulations that describe long distance ballooning intractable. As an example, for some ballooning events, it may be desirable to simulate a meteorological pressure system for which the relevant length scale of the pressure system would be on the order of tens to hundreds of kilometers. In contrast, the relevant length scale of the smallest vortices or eddies may be on the order of one millimeter and the relevant length scale of the spider itself may be on the order of hundreds of microns.

In terms of temporal resolution, ballooning events may take place over the time scale of days, while transient flows that affect ballooning may occur on the scale of milliseconds. Brute force simulation of such a problem would require on the order of $10^{24}$ spatial grid points and $10^{8}$ time steps. Highly efficient, adaptive, and parallelized algorithms that can refine spatial and temporal scales are required to handle this challenge. Scaling laws evaluating the relevant and dominant features required for such modeling would also be critical.

There are a variety of ways in which detailed fluid-structure interaction modeling could help us understand the dynamics of the silk dragline and the ballooning behavior of spiders. Predictive multiscale and multiphysics models could allow us to determine if spiders prefer to take off in certain conditions such as updrafts or within rolling eddies. These models may also help us understand other aspects of ballooning such as turbulence sensing during the tiptoeing behavior and how flow structures may be utilized to enable very long distance dispersal.

In addition to improved numerical methods and mathematical models describing spider ballooning, additional experimental data are needed to guide and validate such models. Wind tunnel studies could be used to examine not only the desired wind speed for takeoff but also the desired wind direction and/or turbulent profile. Spatial and temporal resolution of dispersal patterns could be used to validate models that couple the elastic dynamics of the dragline with complicated flow structures. 
Visualization of the dynamics of the silk dragline in the air could aid in the selection of the appropriate dragline model.

By combining modeling and experimental approaches, a host of possible questions could be explored, including:

- Is elasticity of the dragline (both in terms of resistance to bending and stretching) important for take-off and flight in turbulent regime?

- Is there an optimal dragline length or wind flow profile for take-off, flight, and settling?

- Why do we see such a narrow range of body sizes in ballooning spiders?

- Why is there a maximum wind speed for take-off?

Addressing such questions would help shed light on an interesting ecological mystery surrounding dispersal that has implications for species distributions, invasion biology, and impacts of climate change.

Acknowledgements This work was conducted as part of the Aerodynamics of Spider Ballooning Working Group at the National Institute for Mathematical and Biological Synthesis (NIMBioS), sponsored by the National Science Foundation (NSF) award DBI-1300426 with additional support from The University of Tennessee, Knoxville. We are grateful to Dr. Anita Layton for organizing the NIMBioS workshop. Additional funding was provided by NSF to KSS (Postdoctoral Research Fellowship 1306883), LAM (CBET 1511427), and AC (Graduate Research Fellowship 201315897), and by the Reed and Edgerton Funds at MIT to LB.

\section{References}

1. D. Ahrens, Meteorology today, Brooks/Cole Press, 2003.

2. F.G. Barth, S. Komarek, J.A.C. Humprhey, and B. Treidler, Drop and Swing Dispersal Behavior of A Tropical Wandering Spider - Experiments and Numerical-model, Journal of Comparative Physiology A-sensory Neural and Behavioral Physiology 169 (1991), no. 3, 313-322.

3. L. Bishop, Meteorological aspects of spider ballooning, Environmental Entomology 19 (1990), no. 5, 1381-1387.

4. J.W.M. Bush and D.L. Hu, Walking on water: Biolocomotion at the interface, Annual Review of Fluid Mechanics 38 (2006), no. 1, 339-369.

5. M.L. Cantabrigian, Some observations concerning the odd turn of some shell snails and the darting of spiders, Philosophical Transactions of the Royal Society of London 4 (1669), 10111016.

6. R. Clift, J.R. Grace, and M.E. Weber, Bubbles, drops, and particles, Academic Press, London, 1978.

7. J. Crank, The mathematics of diffusion, Oxford University Press, Oxford, 1975.

8. C.R. Darwin, Journal of researches into the natural history and geology of the countries visited during the voyage of H.M.S. Beagle round the world, under the Command of Capt. Fitz Roy, R.N., 2nd ed., John Murray, London, 1845.

9. J.W. Deardorff, A numerical study of three-dimensional turbulent channel flow at large reynolds numbers, Journal of Fluid Mechanics 41 (1970), 453-480.

10. H. Dingle, Migration : The Biology of Life on the Move: The Biology of Life on the Move, Oxford University Press, USA, 1996.

11. S.F. Dobson, Competition for mates and predominant juvenile male dispersal in mammals, Animal Behaviour 30 (1982), no. 4, 1183-1192. 
12. P. Drazin and W. Reid, Hydrodynamic stability, Cambridge, Cambridge University Press, 1981.

13. E. Duffey, Aerial dispersal in a known spider population, Journal of Animal Ecology 25 (1956), no. 1, 85-111.

14. W.G. Eberhard, How spiders initiate airborne lines, Journal of Arachnology 15 (1987), 1-9.

15. R.F. Foelix, Biology of Spiders, Harvard University Press, Cambridge, Mass., 1982.

16. B_ Biology of Spiders, 3 ed., Oxford University Press, Oxford, UK, 2011.

17. J. Gatesy, C. Hayashi, D. Motriuk, J. Woods, and R. Lewis, Extreme diversity, conservation, and convergence of spider silk fibroin sequences, Science 291 (2001), no. 5513, 2603-2605.

18. T.B. Gatski and J.-P. Bonnet, Compressibility, turbulence and high speed flow, Elsevier, Amsterdam, 2009.

19. P.W. Gorham, Ballooning Spiders: The Case for Electrostatic Flight, ArXiv (2013), 1309.4731v1 [physics.bio-ph].

20. J.M. Gosline, P.A. Guerette, C.S. Ortlepp, and K.N. Savage, The mechanical design of spider silks: from fibroin sequence to mechanical function, Journal of Experimental Biology 202 (1999), no. 23, 3295-3303.

21. M.H. Greenstone, Ballooning frequency and habitat predictability in two wolf spider species ( lycosidae : Pardosa), The Florida Entomologist 65 (1982), no. 1, 83-89.

22. _ Meteorological determinants of spider ballooning: the roles of thermals vs. the vertical windspeed gradient in becoming airborne, Oecologia 84 (1990), no. 2, 164-168.

23. J. L. Gressitt, Biogeography and ecology of land arthropods of antarctica, 1965, pp. 431-490.

24. J. Happel and H. Brenner, Low Reynolds number hydrodynamics: with special applications to particulate media, The Hague; Boston: M. Nijhoff; Hingham, MA, USA, 1983.

25. A. Hedenström, A general law for animal locomotion?, Trends in Ecology and Evolution 19 (2004), no. 5, 217-219.

26. G. Houghton, The behaviour of particles in a sinusoidal velocity field, Proceedings of the Royal Society A: Mathematical, Physical and Engineering Sciences 272 (1963), no. 1348, $33-43$.

27. J.A.C. Humphrey, Fluid mechanic constraints on spider ballooning, Oecologia (1987), 469477.

28. K. Kronenberger and F. Vollrath, Spiders spinning electrically charged nano-fibres., Biology letters 11 (2015), no. 1, 20140813.

29. H. Oertel, Prandtl's essentials of fluid mechanics, New York: Springer, 2004.

30. A.E. Pusey, Sex-biased dispersal and inbreeding avoidance in birds and mammals., Trends in ecology \& evolution 2 (1987), no. 10, 295-9.

31. A.M. Reynolds, D.A. Bohan, and J.R. Bell, Ballooning dispersal in arthropod taxa with convergent behaviours: dynamic properties of ballooning silk in turbulent flows., Biology letters 2 (2006), no. 3, 371-3.

32. Ballooning dispersal in arthropod taxa: conditions at take-off., Biology letters 3 (2007), no. 3, 237-40.

33. P. Sagaut, Large eddy simulation for incompressible flows, third ed. ed., Springer, 2006.

34. J.M. Schneider, J. Roos, Y. Lubin, and J.R. Henschel, Dispersal of Stegodyphus Dumicola (Araneae, Eresidae): They Do Balloon After All!, Journal of Arachnology 29 (2001), no. 1, $114-116$.

35. J. Smagorinsky, General circulation experiments with the primitive equations, Monthly Weather Review 91 (1963), no. 3, 99-164.

36. R.B. Suter, Ballooning in spiders: results of wind tunnel experiments, Ethology Ecology \& Evolution 3 (1991), no. 1, 13-25.

37. Ballooning: Data From Spiders in Freefall Indicate the Importance of Posture, Journal of Arachnology 20 (1992), 107-113.

38. _ An aerial lottery: The physics of ballooning in a chaotic atmosphere, The journal of Arachnology (1999), 281-293.

39. G.K. Taylor, R.L. Nudds, and A.L.R. Thomas, Flying and swimming animals cruise at a Strouhal number tuned for high power efficiency., Nature 425 (2003), no. 6959, 707-711. 
40. H. Tennekes and J.L. Lumley, A first course in turbulence, The MIT Press, Cambridge, MA, 1972.

41. C.F.G. Thomas, P. Brain, and P.C. Jepson, Aerial activity of linyphiid spiders: Modelling dispersal distances from meteorology and behaviour, Journal of Applied Ecology 40 (2003), no. 5, 912-927.

42. C.F.G. Thomas, E.H.A. Hol, and J.W. Everts, Modelling the diffusion component of dispersal during recovery of a population of linyphiid spiders from exposure to an insecticide, Functional Ecology 4 (1990), no. 3, 357-368.

43. H.F. Vugts and W.K.R.E. Van Wingerden, Meteorological aspects of aeronautic behaviour of spiders, Oikos 27 (1976), no. 3, 433-444.

44. G.S. Weyman, A review of the possible causative factors and significance of ballooning in spiders, 1993, pp. 279-291.

45. L__ Laboratory studies of the factors stimulating ballooning behavior by linyphiid spiders (Araneae, Linyphiidae), Journal of Arachnology (1995), no. 25, 75-84.

46. W. Wickler and U. Seibt, Aerial dispersal by ballooning in adult Stegodyphus mimosarum, Naturwissenschaften 73 (1986), no. 10, 628-629.

47. D.C. Wilcox, Turbulence modeling for cfd, DCW Industries, La Canada, CA, 2006.

48. J.O. Wolff, What Is the Role of Adults in Mammalian Juvenile Dispersal?, Oikos 68 (1993), no. $1,173-176$. 